\title{
A Facile Strategy for the Functionalization of Boron Nitride Nanotubes with Pd Nanoparticles
}

\author{
Yuanlie Yu, ${ }^{1}$ Hua Chen, ${ }^{1}$ and Yun Liu ${ }^{2}$ \\ ${ }^{1}$ Centre for Advanced Microscopy, Australian National University, Canberra, ACT 0200, Australia \\ ${ }^{2}$ Research School of Chemistry, Australian National University, Canberra, ACT 0200, Australia \\ Correspondence should be addressed to Hua Chen; hua.chen@anu.edu.au
}

Received 7 October 2014; Accepted 17 November 2014

Academic Editor: Mengnan Qu

Copyright (C) 2015 Yuanlie Yu et al. This is an open access article distributed under the Creative Commons Attribution License, which permits unrestricted use, distribution, and reproduction in any medium, provided the original work is properly cited.

\begin{abstract}
A facile in situ fabrication of palladium nanoparticles decorated boron nitride nanotubes (Pd-BNNTs) is described. The decoration of BNNTs was carried out by the self-regulated reduction of palladium chloride $\left(\mathrm{PdCl}_{2}\right)$ with the aid of sodium dodecyl sulfate (SDS). During the preparation process, the surfactant SDS plays a dual role: it aids the dispersibility of BNNTs and produces the reductant of $\mathrm{CH}_{3}\left(\mathrm{CH}_{2}\right)_{10} \mathrm{CH}_{2} \mathrm{OH}$. Then the $\mathrm{CH}_{3}\left(\mathrm{CH}_{2}\right)_{10} \mathrm{CH}_{2} \mathrm{OH}$ can reduce $\mathrm{Pd}^{2+}$ to form $\mathrm{Pd}$ nanoparticles on the surface of BNNSs. The as-prepared Pd-BNNTs were characterized by field emission scanning electron microscopy, transmission electron microscopy, X-ray energy dispersive spectroscopy, and Fourier transform infrared spectroscopy. The results show that the Pd nanocrystalline particles can be deposited onto the BNNTs surface via this simple route. This approach constitutes a basis for the assembly and integration of nanoscale materials onto BNNTs and puts a light on the potential application of the BNNTs in electronic, catalysis, and hydrogen storage fields.
\end{abstract}

\section{Introduction}

Boron nitride nanotubes (BNNTs) have recently attracted increased attention due to their various potential applications in electronic, mechanical, and optical devices. Structurally similar to carbon nanotubes (CNTs), BNNTs exhibit excellent mechanical [1-3] and thermal [4-6] properties. Despite these similarities, BNNTs exhibit excellent chemical stability and high resistance to oxidation at high temperature [7]. BNNTs are uniform semiconductors with a bandgap of $5.5 \mathrm{eV}$, basically independent of tube chirality and diameters [8], while CNTs exhibit variable metallic and semiconducting characteristics. In addition, recent research has also demonstrated that BNNTs can be used as room temperature hydrogen storage media [9], humidity sensor devices [10], and oil/water separation materials [11]. Considering the abovementioned properties, BNNTs have become very attractive for innovative applications in various fields of science and technology, especially in hazardous and high temperature environments. However, the insolubility in common solvents and good insulating property also limit their applications. Further modification of BNNTs is therefore necessary for the utilization of BNNT-based materials for novel sensory, electronic, catalytic, and biomedical applications. For example, the multiwalled BNNTs modified with long alkyl chains are soluble in many organic solvents [12]. And subsequent cathodoluminescence and UV-Vis absorption tests show that long alkyl chains can induce drastic changes in the band structure of BNNTs [12]. The Au decoration on the surface of BNNTs significantly enhanced their field emission current densities [13]. Additionally, the introduction of rare-earth element into the walls of BNNTs can result in broad and tunable light emission $[14,15]$, making them a potential candidate in nanosized lighting sources and nanospectroscopy. Yet it is difficult to directly decorate the BNNTs with metal nanoparticles by chemical route due to the good chemical inertness of the BNNTs. At present, the synthesis of metal nanoparticles decorated BNNTs is mainly realized by physical vapour deposition (PVD) [13] or a complicated chemical route [16]. Although metal nanoparticles with uniform size can be obtained by PVD, the deposition only occurs on the top surface of bulk BNNTs samples facing the metal target. And, for previous chemical route, the surface of the BNNTs and metal nanoparticles needs to be functionalized with 
organic molecules, which is fairly complicated. Therefore, simpler and effective methods are required to prepare these metal nanoparticles decorated BNNTs.

In this work, a facile in situ preparation of Pd-BNNTs is developed. The process was carried out by self-regulated reduction of $\mathrm{PdCl}_{2}$ with the aid of SDS. The method provides a basis for the assembly and integration of nanoscale materials onto the BNNTs. In addition, Pd is an important transition metal in catalysis due to their high hydrogen solubility, diffusivity, and corrosion resistance $[17,18]$ and it can also be used as hydrogen storage media [19] for its high affinity for hydrogen. That is, the introduction of Pd may improve the catalytic activity and hydrogen adsorption property of the BNNTs, making them potential candidate in these fields.

\section{Experimental Procedure}

The BNNTs were synthesized by a ball milling and subsequent high temperature annealing process [13-15]. Typically, the amorphous boron powder was first milled in a planetary mill with a stainless steel bowl and several balls for $50 \mathrm{~h}$ at a rotation speed of $300 \mathrm{rpm}$. The weight ratio of the milling balls to the boron powder was $50: 1$. Then, the milled boron powder was annealed in a tube furnace at $1100^{\circ} \mathrm{C}$ for $2 \mathrm{~h}$ under a $\mathrm{H}_{2} / \mathrm{N}_{2}\left(15 \% \mathrm{H}_{2}\right)$ gas flow $(100 \mathrm{~mL} / \mathrm{min})$. Then, $0.5 \mathrm{mg}$ of as-synthesized BNNTs was suspended in $2 \mathrm{~mL}$ of $0.05 \mathrm{M}$ aqueous SDS solution and ultrasonicated for $30 \mathrm{~min}$. Subsequently, $1 \mathrm{~mL}$ of $\mathrm{PdCl}_{2}$ saturated solution $\left(20^{\circ} \mathrm{C}\right)$ was added to the BNNTs/SDS dispersion and the mixture was refluxed for $6 \mathrm{~h}$ at $130 \pm 1^{\circ} \mathrm{C}$. After cooling down to room temperature naturally, the reaction mixture was filtered with $0.45 \mu \mathrm{m}$ membrane filter, washed several times with distilled water to remove the excess surfactant, and then redissolved in ethanol.

Zeiss UltraPlus analytical field emission scanning electron microscopy (FESEM) was used for morphology investigation. X-ray energy dispersive spectroscopy (EDS) attached to the Zeiss UltraPlus analytical FESEM was used to examine the components. Philips CM300 high-resolution transmission electron microscopy (HRTEM) was employed to probe the interior construction of the as-prepared BNNTs and PdBNNTs. The X-ray diffraction spectrum was collected with BRUKER-AXS X-ray diffractometer (XRD) with monochromatic $\mathrm{Cu} \mathrm{K} \alpha$ radiation. The bonding information of BNNTs was identified with PerkinElmer Fourier transform infrared spectroscopy (FTIR).

\section{Results and Discussion}

As shown in Figure 1(a), the typical XRD pattern of the as-prepared BNNTs shows three peaks (marked with black rhombus " $\$$ ") at the d-spacing of $3.36,2.15$, and 2.06 which can be assigned to (002), (100), and (101) planes of hexagonal boron nitride structure (JCPDF card number 73-2095) [20]. The rest peak marked with hollow rhombus $(\nabla)$ can be identified as (110) plane of $\alpha$-Fe, which was introduced in the ball milling process and used as catalyst in the BNNTs growth process. In addition, two IR absorption regions can be obviously distinguished at $805 \mathrm{~cm}^{-1}$ and $1385 \mathrm{~cm}^{-1}$ in the FTIR spectrum (Figure 1(b)), corresponding to the out-ofplane radial buckling $(\mathrm{R})$ mode where boron and nitrogen atoms are moving radially inward or outward and transverse optical (TO) mode of h-BN sheets that vibrate along the longitudinal or tube axis of BNNTs, respectively [21, 22]. Figures $1(\mathrm{c})$ and $1(\mathrm{~d})$ show the typical FESEM and TEM images and EDS spectrum of the as-prepared BNNTs. The FESEM image (Figure 1(c)) reveals that pure products were formed on the sample surfaces, which possess a high density of onedimensional structures with relatively uniform diameters in the range of 100-300 $\mathrm{nm}$. The inset EDS spectrum confirms that the main components composed of the nanotubes are boron and nitride. Further examination carried out by TEM analysis (Figure $1(\mathrm{~d})$ ) indicates that the BNNTs are formed by repeated cup-like structures with void interior. The intrinsic plane spacing of the walls is determined to be $0.34 \mathrm{~nm}$ in terms of the HRTEM images (inset in Figure $1(\mathrm{~d})$ ), which is well matched with that of h-BN (002) plane.

As described in Figure 2, the in situ decoration of BNNTs with $\mathrm{Pd}$ nanoparticles is realized by refluxing a dispersion of BNNTs in an aqueous solution of $\mathrm{SDS} / \mathrm{PdCl}_{2}$ for $6 \mathrm{~h}$ (details can be seen in Experimental Procedure section). During the whole preparation process, the surfactant SDS plays a dual role. Firstly, as the surfactant, a great deal of SDS molecules will be absorbed on the surface of the BNNSs thus aiding the dispersion of BNNTs in water as shown in Figure 2. Secondly, SDS acts as a reactant to participate in the oxidation-reduction reaction. During the reflux process, $\mathrm{CH}_{3}\left(\mathrm{CH}_{2}\right)_{10} \mathrm{CH}_{2} \mathrm{OH}$ was generated by the reaction of SDS with $\mathrm{H}_{2} \mathrm{O}$ (reaction (1) in Figure 3), and then the $\mathrm{Pd}^{2+}$ ions that had diffused into the core of the micelle were reduced by the $\mathrm{CH}_{3}\left(\mathrm{CH}_{2}\right)_{10} \mathrm{CH}_{2} \mathrm{OH}$ to form $\mathrm{Pd}$ atoms (reaction (2) in Figure 3) [23]. With the increase in the reduced Pd atoms, they agglomerated together on the BNNTs' surface to form Pd nanoparticles. After separating and carefully rinsing, the residual organic was removed and Pd-BNNTs were achieved. Figure 4(a) shows a typical SEM image of the Pd nanoparticles decorated BNNT, where Pd nanoparticles can be clearly seen attached on the surface of BNNT. EDS analysis (inset in Figure 4(a)) confirmed that the attached nanoparticles are indeed palladium. Figures 4(b) and 4(c) present the TEM micrographs of pristine and Pd nanoparticles decorated BNNTs, respectively. Comparing Figure 4(c) with Figure 4(b), the dark spots particles can be clearly seen on the surface of Pd nanoparticles decorated BNNT. Inset in Figure 4(c) is a typical HRTEM image of the Pd nanoparticles attached on the surface of BNNTs. The lattice constant of the nanoparticles is calculated to be $0.197 \mathrm{~nm}$, which is the same as the (200) plane distance of the cubic Pd crystal structure [24], demonstrating that the Pd nanoparticles are successfully deposited on the surface of the BNNTs.

\section{Conclusions}

It has been demonstrated that Pd-BNNTs can be prepared by the self-regulated reduction of $\mathrm{PdCl}_{2}$ with the aid of SDS. 

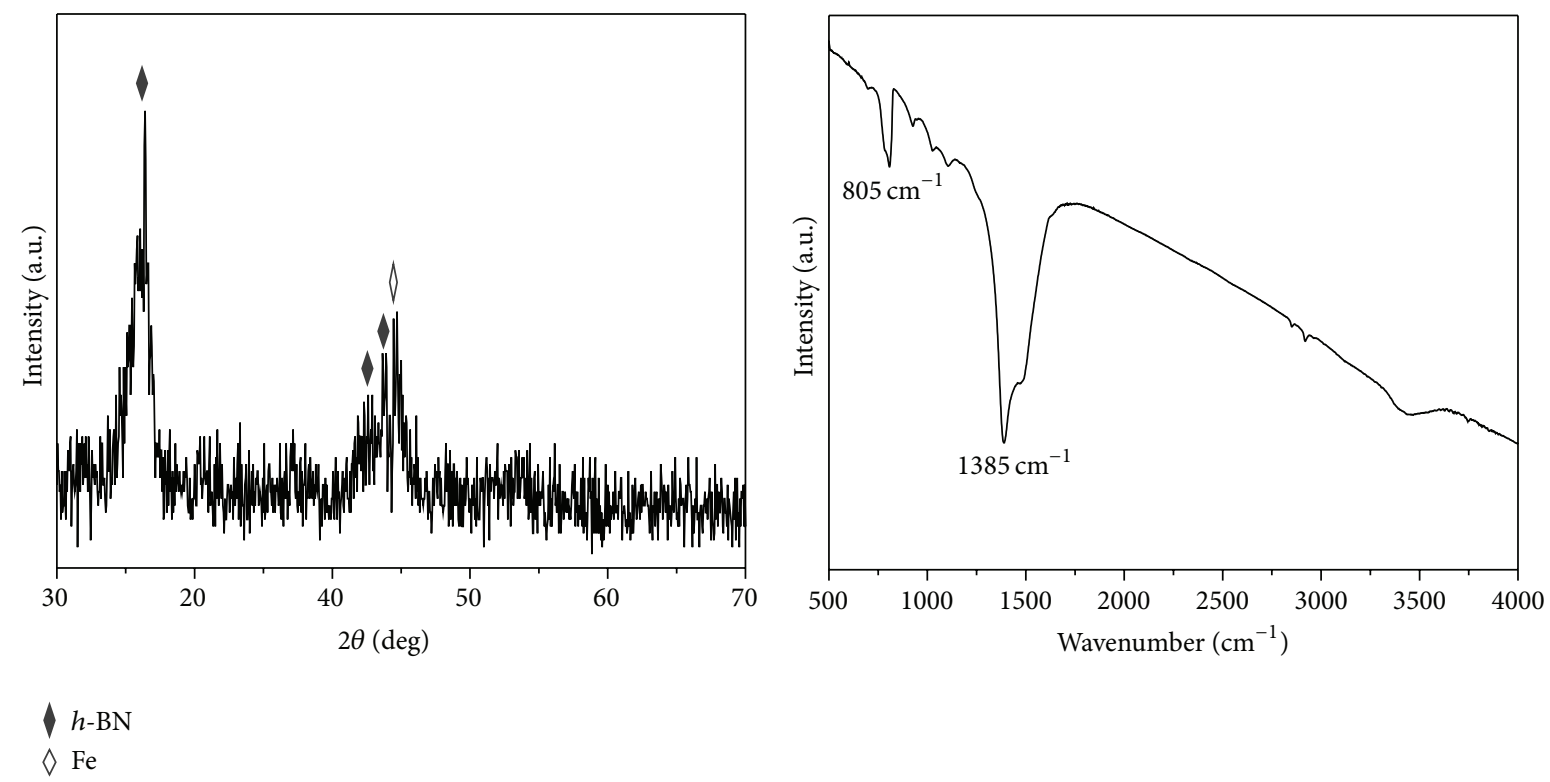

(a)

(b)

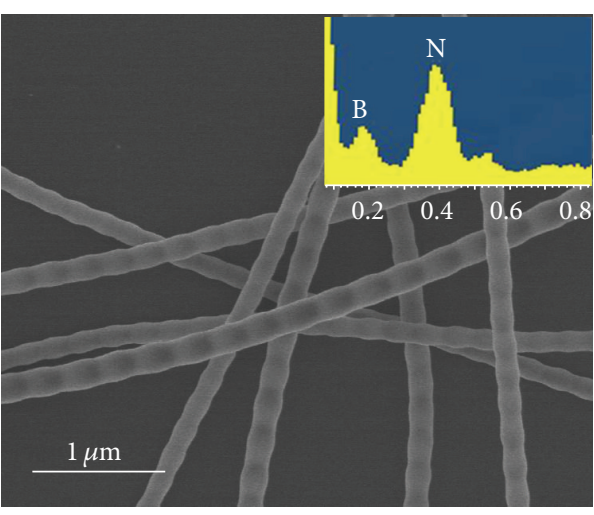

(c)

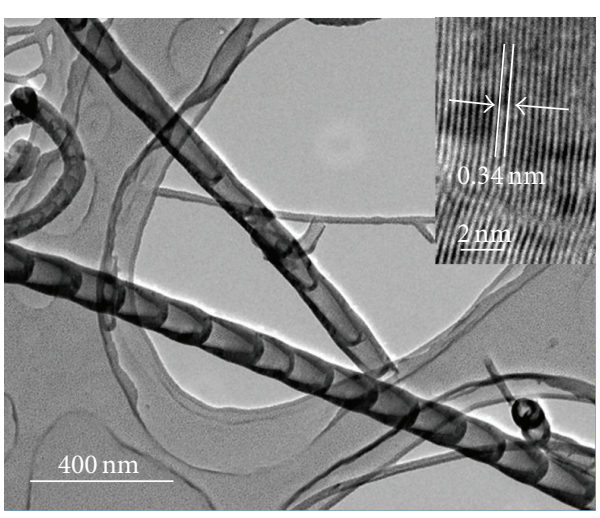

(d)

FIGURE 1: (a) The XRD pattern and (b) the FTIR spectrum of the BNNTs; (c) and (d) the FESEM image (inset is the EDS spectrum) and the TEM image (inset is the HRTEM image) of the as-synthesized BNNTs.

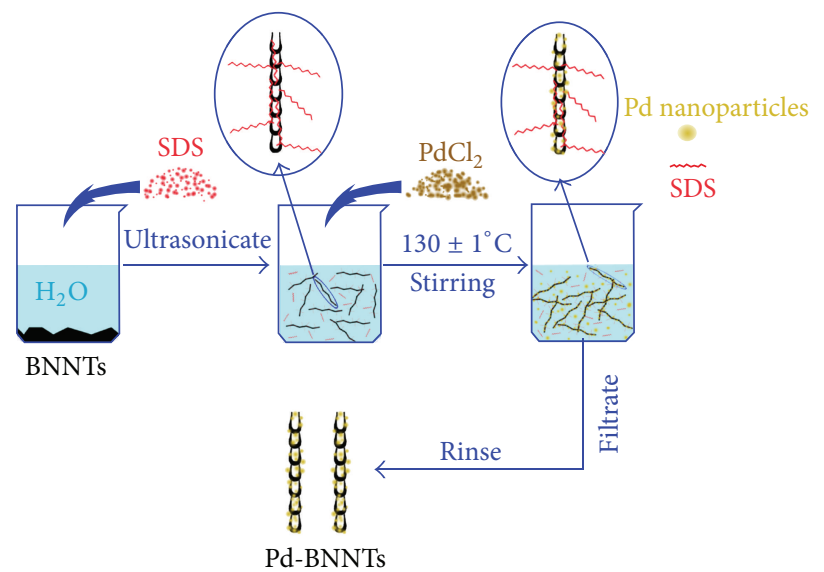

FIgURE 2: The process for the decoration of BNNTs with Pd nanoparticles. 


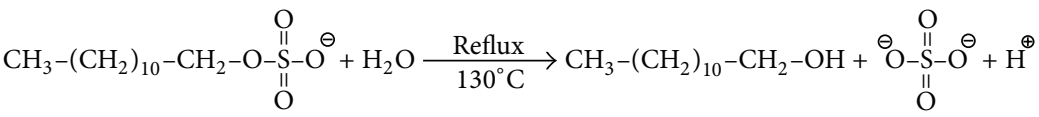

$$
\begin{aligned}
& \mathrm{CH}_{3}-\left(\mathrm{CH}_{2}\right)_{10}-\mathrm{CH}_{2}-\mathrm{OH}+\mathrm{Pd}^{2 \oplus}+\mathrm{H}_{2} \mathrm{O} \underset{130^{\circ} \mathrm{C}}{\stackrel{\text { Reflux }}{\longrightarrow}} \mathrm{CH}_{3}-\left(\mathrm{CH}_{2}\right)_{10}-\mathrm{CH}_{2}-\mathrm{OH}+\mathrm{Pd}^{0}+2 \mathrm{H}^{\oplus}
\end{aligned}
$$

FIGURE 3: The reactions during the reflux process.

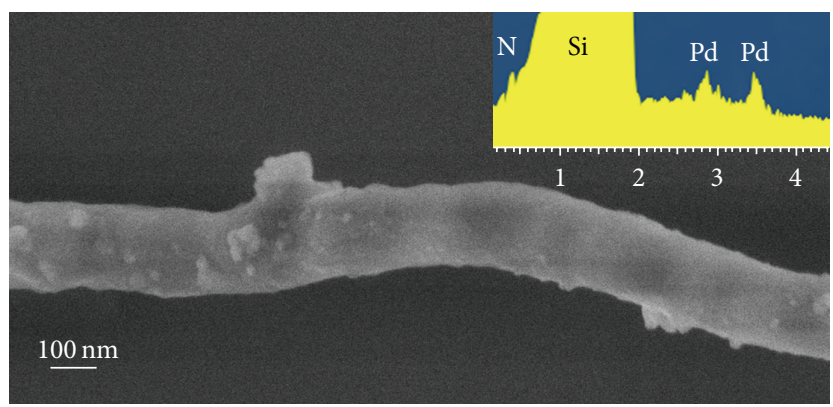

(a)

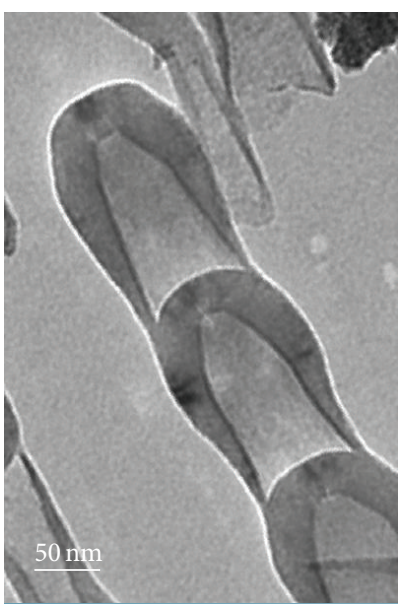

(b)

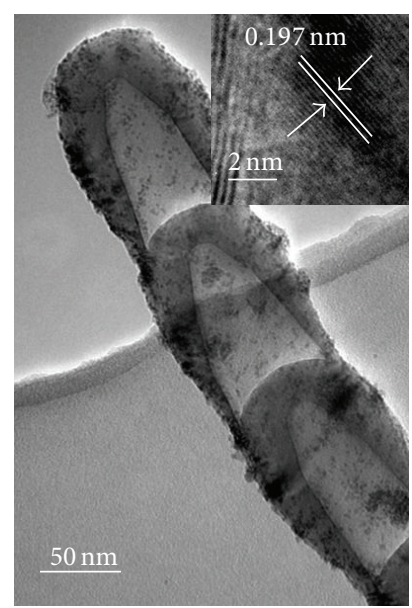

(c)

FIGURE 4: (a) FESEM image of the Pd-BNNTs (inset is the corresponding EDS spectrum); (b) and (c) the typical TEM images of pristine BNNTs and BNNTs with Pd nanoparticles (inset is the HRTEM image).

During the preparation process, the surfactant SDS plays a very important role in the dispersibility of BNNTs and reduction of $\mathrm{Pd}^{2+}$ to form $\mathrm{Pd}$ nanoparticles. The characterization results indicate that $\mathrm{Pd}$ nanocrystalline particles can be successfully deposited on the BNNTs surface via this simple wet chemical route, which constitutes a basis for the assembly and integration of nanoscale materials onto the BNNTs. The good activity catalysis of Pd combined with the excellent properties of BNNTs may make the Pd-BNNTs be potential candidate in the application of electronic and catalysis fields.

\section{Conflict of Interests}

The authors declare that there is no conflict of interests regarding the publication of this paper.

\section{Acknowledgments}

The authors acknowledge the financial support from the Australian Microscopy and Microanalysis Research Facility (AMMRF) and from the Australian Research Council in the form of the Discovery Projects.

\section{References}

[1] N. G. Chopra and A. Zettl, "Measurement of the elastic modulus of a multi-wall boron nitride nanotube," Solid State Communications, vol. 105, no. 5, pp. 297-300, 1998.

[2] A. P. Suryavanshi, M.-F. Yu, J. Wen, C. Tang, and Y. Bando, "Elastic modulus and resonance behavior of boron nitride nanotubes," Applied Physics Letters, vol. 84, no. 14, pp. 25272529, 2004. 
[3] D. Golberg, P. M. F. J. Costa, O. Lourie et al., "Direct force measurements and kinking under elastic deformation of individual multiwalled boron nitride nanotubes," Nano Letters, vol. 7, no. 7, pp. 2146-2151, 2007.

[4] L. Wirtz and A. Rubio, " $A b$ initio calculations of the lattice dynamics of boron nitride nanotubes," Physical Review B, vol. 68, no. 4, Article ID 045425, 2003.

[5] D. Sanchez-Portal and E. Hernandez, "Vibrational properties of single-wall nanotubes and monolayers of hexagonal BN," Physical Review B, vol. 66, no. 23, Article ID 235415, pp. 1-12, 2002.

[6] V. N. Popov, "Lattice dynamics of single-walled boron nitride nanotubes," Physical Review B, vol. 67, no. 8, Article ID 085408, 6 pages, 2003.

[7] Y. Xiao, X. H. Yan, J. X. Cao, J. W. Ding, Y. L. Mao, and J. Xiang, "Specific heat and quantized thermal conductance of singlewalled boron nitride nanotubes," Physical Review B, vol. 69, no. 20, Article ID 205415, 2004.

[8] A. Rubio, J. L. Corkill, and M. L. Cohen, "Theory of graphitic boron nitride nanotubes," Physical Review B, vol. 49, no. 7, pp. 5081-5084, 1994.

[9] R. Z. Ma, Y. Bando, H. W. Zhu, T. Sato, C. L. Xu, and D. H. $\mathrm{Wu}$, "Hydrogen uptake in boron nitride nanotubes at room temperature," Journal of the American Chemical Society, vol. 124, no. 26, pp. 7672-7673, 2002.

[10] Y. Yu, H. Chen, Y. Liu, L. H. Li, and Y. Chen, "Humidity sensing properties of single Au-decorated boron nitride nanotubes," Electrochemistry Communications, vol. 30, pp. 29-33, 2013.

[11] Y. L. Yu, H. Chen, Y. Liu, V. Craig, L. H. Li, and Y. Chen, "Superhydrophobic and superoleophilic boron nitride nanotubecoated stainless steel meshes for oil and water separation," Advanced Materials Interfaces, vol. 1, no. 1, Article ID 1300002, pp. 1-5, 2014.

[12] C. Zhi, Y. Bando, C. Tang et al., "Covalent functionalization: towards soluble multiwalled boron nitride nanotubes," Angewandte Chemie International Edition, vol. 44, no. 48, pp. 79327935, 2005.

[13] H. Chen, H. Zhang, L. Fu et al., "Nano Au-decorated boron nitride nanotubes: conductance modification and fieldemission enhancement," Applied Physics Letters, vol. 92, no. 24, Article ID 243105, 2008.

[14] H. Chen, Y. Chen, C. P. Li et al., "Eu-doped boron nitride nanotubes as a nanometer-sized visible-light source," Advanced Materials, vol. 19, no. 14, pp. 1845-1848, 2007.

[15] H. Chen, Y. Chen, and Y. Liu, "Cathodoluminescence of boron nitride nanotubes doped by ytterbium," Journal of Alloys and Compounds, vol. 504, supplement 1, pp. S353-S355, 2010.

[16] H. Chen, Y. Chen, Y. Liu et al., "Rare-earth doped boron nitride nanotubes," Materials Science and Engineering: B, vol. 39, no. 40, pp. 189-192, 2008.

[17] T. Sainsbury, T. Ikuno, D. Okawa, D. Pacilé, J. M. J. Fréchet, and A. Zettl, "Self-assembly of gold nanoparticles at the surface of amine- and thiol-functionalized boron nitride nanotubes," Journal of Physical Chemistry C, vol.111, no. 35, pp. 12992-12999, 2007.

[18] B. M. Trost, J. Xu, and T. Schmidt, "Palladium-catalyzed decarboxylative asymmetric allylic alkylation of enol carbonates," Journal of the American Chemical Society, vol. 131, no. 51, pp. 18343-18357, 2009.

[19] S. Yu, U. Welp, L. Z. Hua, A. Rydh, W. K. Kwok, and H. H. Wang, "Fabrication of palladium nanotubes and their application in hydrogen sensing," Chemistry of Materials, vol. 17, no. 13, pp. 3445-3450, 2005.

[20] Q. Qian, J. Wang, Y. Gu et al., "Convenient synthesis of Fe-filled boron nitride nanotubes by SHS method," Materials Letters, vol. 65, no. 5, pp. 866-868, 2011.

[21] E. Borowiak-Palen, T. Pichler, G. G. Fuentes et al., "Infrared response of multiwalled boron nitride nanotubes," Chemical Communications, vol. 9, no. 1, pp. 82-83, 2003.

[22] J. L. Wang, C. H. Lee, Y. Bando, D. Golberg, and Y. K. Yap, "B-C$\mathrm{N}$ nanotubes and related nanostructures," in Multiwalled Boron Nitride Nanotubes: Growth, Properties, and Applications, vol. 6 of Lecture Notes in Nanoscale Science and Technology, chapter 2, pp. 23-44, Springer, New York, NY, USA, 2009.

[23] C. L. Lee, C. C. Wan, and Y. Y. Wang, "Synthesis of metal nanoparticles via self-regulated reduction by an alcohol surfactant," Advanced Functional Materials, vol. 11, no. 5, pp. 344-347, 2011.

[24] F. Ksar, G. Surendran, L. Ramos et al., "Palladium nanowires synthesized in hexagonal mesophases: application in ethanol electrooxidation," Chemistry of Materials, vol. 21, no. 8, pp. 16121617, 2009. 

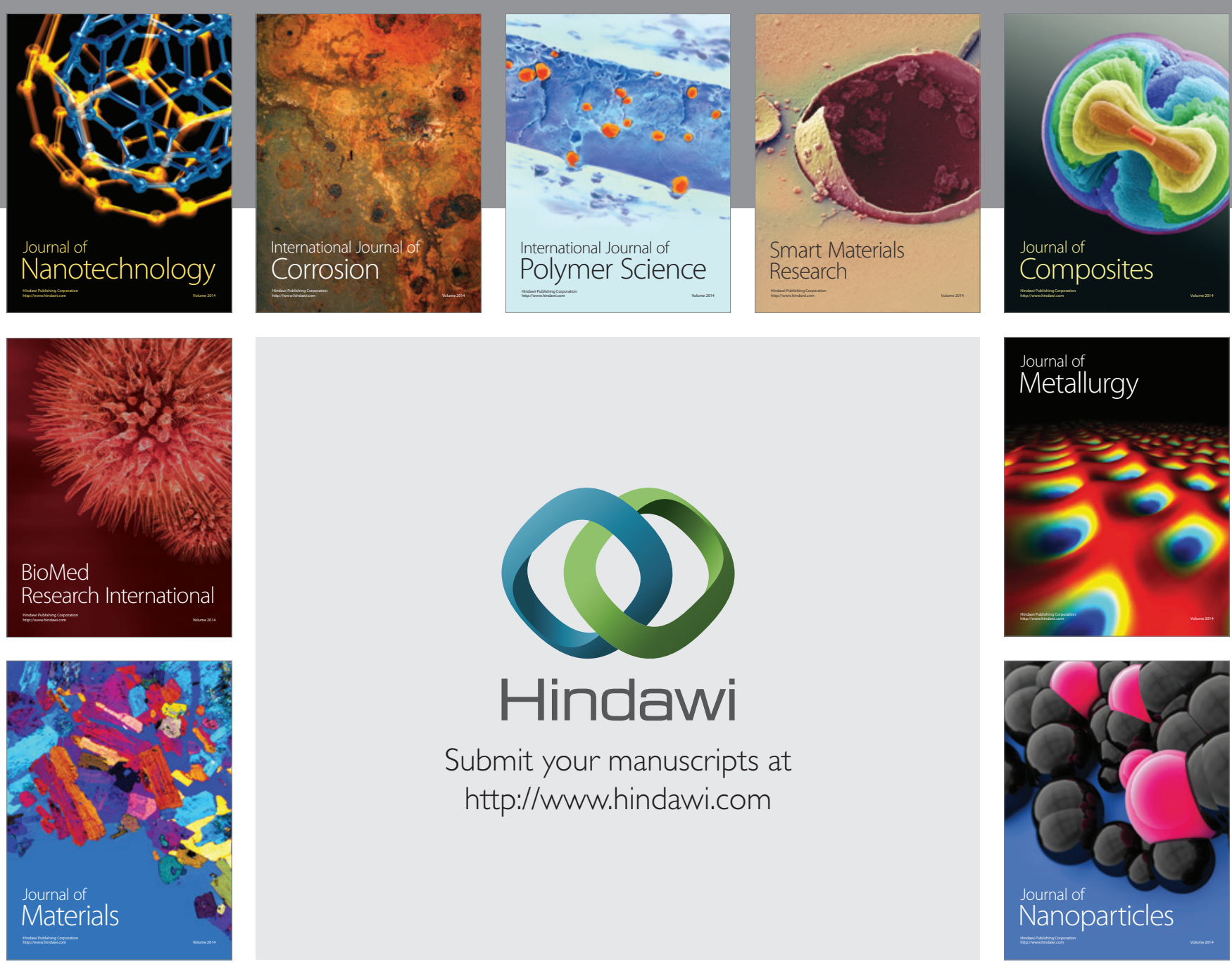

Submit your manuscripts at http://www.hindawi.com
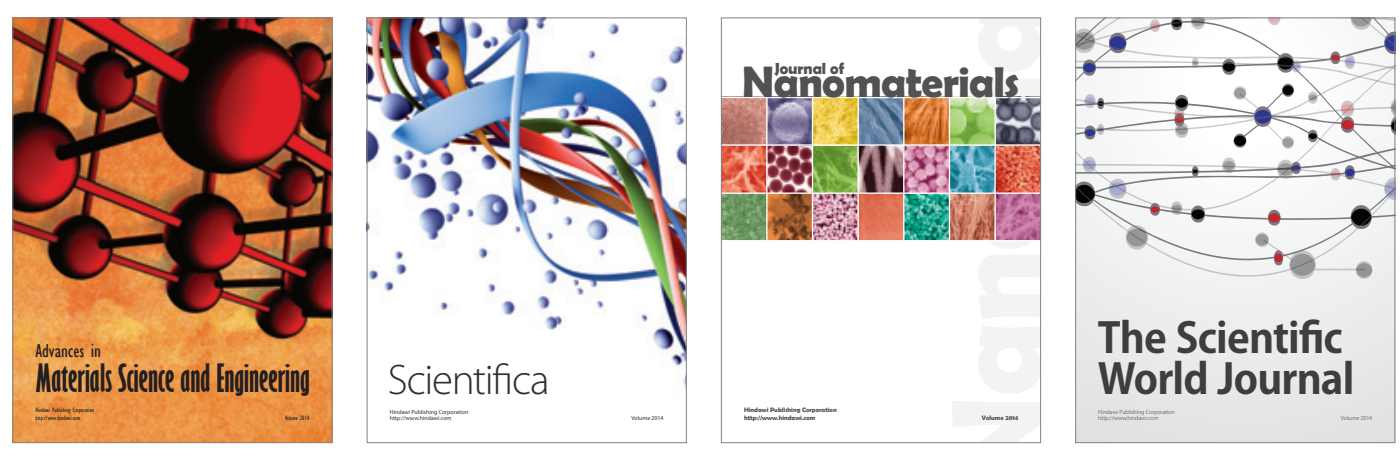

\section{The Scientific World Journal}
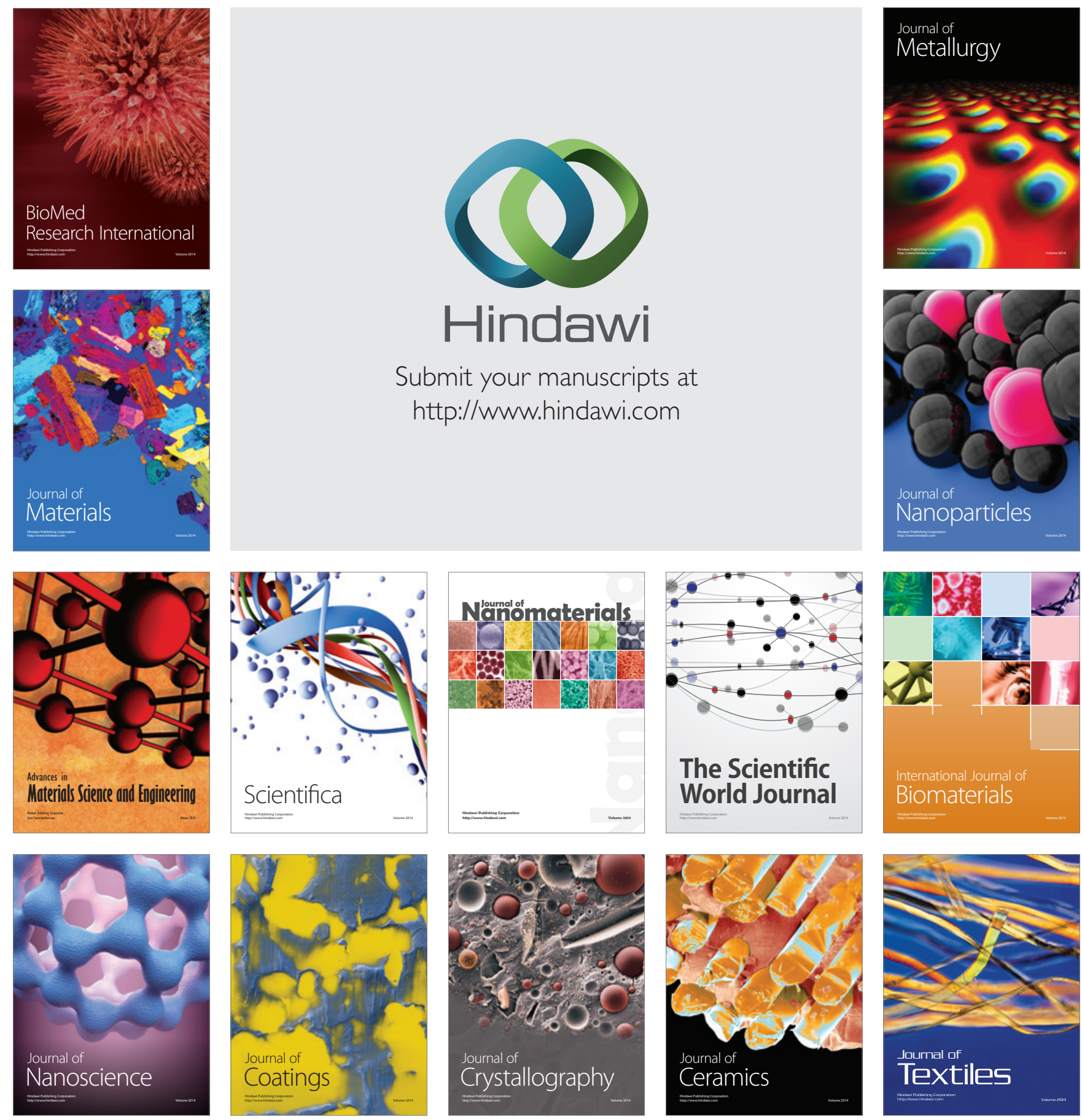